\title{
CANINE BACTERIAL ZOONOSIS
}

\author{
Mohamed M S Gaballa and Salma A Shoulah² \\ 'Department of Pathology, Faculty of Veterinary Medicine, Benha University, Egypt \\ ${ }^{2}$ Department of Animal Medicine (Infectious Diseases), Faculty of Veterinary Medicine, Benha University, Egypt \\ *Corresponding author: mohamed.gaballah@fvtm.bu.edu.eg
}

\section{INTRODUCTION}

The degree to which people find pleasure in the companionship of dogs is evident by the number of household dogs in the human community. With the everincreasing ownership of dogs, the likelihood of infection with dog bacteria and other pathogenic agents is prevalent among canines and has steadily increased as well. Dogs are a common host species to a number of zoonotic diseases, which have a significant impact on public health. While some of these diseases are widely distributed but mild in nature, others, mostly caused by bacterial agents, can cause serious or even fatal illness. To compound matters, antimicrobial use (and abuse) for treating both human and zoonotic illness has led to antimicrobial resistance. Such antimicrobial resistance inevitably leads to 'epidemics' of resistant bacterial infections, from which dogs are not exempted. The spread of certain multidrugresistant bacteria from people to their dogs represents a fascinating example of the one-medicine principle. This chapter focuses on bacterial zoonoses linked to dogs. Evidently, clear guidance, stemming from current knowledge of the epidemiology, pathobiology and diagnosis of bacterial dog-related human diseases is needed in order to provide dog owners with objective knowledge of best practices pertaining to dog care.

\section{Salmonellosis}

\section{Overview}

Salmonellosis is a bacterial disease caused by a number of bacteria of the genus Salmonella, which includes over 2300 serovars; often transmitted to humans through the fecal-oral route. The infection leads to enteric and multisystem disease in both humans and animals. Human salmonellosis is an essential zoonosis with broad economic and public health implications. While salmonellosis is most frequently caused by foodborne infection, Salmonella's zoonotic transmission from dogs has also been documented (Leonard 2014).

\section{Etiology}

Salmonella spp. are ubiquitous opportunistic, usually motile, aerobic, and facultative anaerobic, non-sporeforming gram-negative rods. They belong to the family Enterobacteriaceae; members of this family are capable of colonizing the gastrointestinal tracts of humans, dogs, a large number of other mammalian species, as well as birds and reptiles. Recent findings suggest that Salmonella is rarely detected in healthy dogs, $0-2.9 \%$ of pet-household dogs and $6.3 \%$ of stray dogs. Detection rates for Salmonella are considerably higher in dogs on a raw-meat diet, with 23 times higher Salmonella shedding, compared with those not fed raw meat. Moreover, in healthy dogs, one single Salmonella contaminated raw meat meal can contribute to fecal shedding of Salmonella for up to one week (Joffe and Schlesinger 2002; Morley et al. 2006; Finley et al. 2007; Tsai et al. 2007; Lefebvre et al. 2008).

\section{Clinico-pathological features}

Adult infected dogs tend to shed Salmonella asymptomatically, while younger, aged and pregnant dogs experience clinical symptoms after 3-to-5-days incubation period. Symptoms typically range from mild self-limited diarrhea to symptoms of severe hemorrhagic gastroenteritis and septicemia. Following the ingestion of contaminated food, Salmonella bind to, colonize and penetrate enterocytes and proliferate both freely and inside macrophages, provoking mucosal necrosis with proprial and submucosal hemorrhage, edema, and leukocytic infiltration. The affected mucosa is typically hyperemic or hemorrhagic, thickened and covered with a red, yellow, or gray exudate. In young animals infected with virulent strains, macrophages may transport Salmonella to the mesenteric lymph nodes and blood vessels, leading to circulatory invasion and septicemia.

Dogs start to become anorexic and develop fever. Diarrhea, in the form of mucoid, watery, or hemorrhagic feces, usually follows. Vomiting, dehydration, and potential hypovolemic shock and septicemia can occur, particularly in severe cases. Once clinical symptoms subside, dogs tend to shed Salmonella for a short period that may extend up to eight weeks in some cases. Pregnant females infected with Salmonella show vaginal discharge, followed by abortion.

In humans, the infection may be asymptomatic. In clinical cases, acute gastro-enterocolitis develops after an incubation period of 4-10 days in children, the elderly, and those who suffer from immunosuppression. Symptoms typically include fever, nausea, abdominal pain, and mucoid diarrhea. Bloody diarrhea is more common in children than in adults. Possible non-gut sequalae include skin lesions, arthritis, and septicemia (Weese and Fulford 2011; Greene 2012; Macpherson et al. 2013; O'Neil 2018). 


\section{Diagnosis}

The standard diagnostic test for Salmonellosis requires early isolation of the organisms from feces after the initial signs of illness. In cases of disseminated disease, bacteria can be isolated from the blood or other sterile body sites. Selective media, such as selenite or tetrathionate broth, are necessary for cultivation. As a means of rapid diagnosis, PCR testing is becoming more popular. Unfortunately, validation of PCR conducted to detect Salmonella is currently lacking, and the efficacy of Salmonella PCR tests is still unclear. MALDI-TOF mass spectrometry and DNA microarrays have recently become additional methods for diagnosis of Salmonella infections (Singhal et al. 2015; Bauerfeind and Krauss 2016).

\section{Therapeutics}

In dogs, there is no antibiotic therapy indicated for uncomplicated cases. However, antibiotic therapy should be prescribed in severe cases or those with extra-intestinal complications and in animals that are chronic carriers for up to 6 weeks after checking for antibiotic resistance. Ampicillin, amoxicillin/clavulanate, fluoroquinolones, 3rd-generation cephalosporin are antibiotics of choice for the treatment of Salmonella infections.

In human, patients who do not belong to a risk category, mild infections need no antimicrobials, with only fluid and electrolyte replacement is required as treatment. In patients at high risk of invasive infection, including neonates, elderly and immunosuppressed persons, antimicrobials, such as ciprofloxacin or trimethoprimsulfamethoxazole, should be administered for 3 to 7 days; also ciprofloxacin may be given for up to 10 days in permanent excretors. However, a high percentage of Salmonella has developed multiple antibiotic resistance and the treatment should only be given according to the antibiogram findings (Wiebe 2015; Bauerfeind and Krauss 2016; O’Neil 2018; Weese and Evason 2019).

\section{Campylobacteriosis}

\section{Overview}

Campylobacteriosis is a widespread bacterial disease that affects both humans and a number of domesticated animal species. It is caused by several bacterial species of the genus Campylobacter (formerly Vibrio). Campylobacter, a major cause of human enteritis, generally colonizes in the gastrointestinal tract of wild and domestic animals and can be isolated from both healthy, as well as diarrhea-affected dogs. Most human cases of Campylobacteriosis are foodborne, typically occurring after the handling or consumption of contaminated, untreated surface water, unpasteurized milk, or undercooked meat. Dogs and other companion animals are also possible sources of human infection, and their true roles may be currently underestimated. Around 6\% of cases of human Campylobacteriosis have been identified to have occurred due to contact with pets (Tenkate and
Stafford 2001; Koene et al. 2004; Adak et al. 2005; Mazick et al. 2006; Rossi et al. 2008).

\section{Etiology}

Campylobacter spp. are motile, slender, spiral or commashaped gram-negative rods that grow best under $\begin{array}{llllllll}\text { microaerophilic } & 5 \% & \mathrm{O}_{2}, & 10 \% & \mathrm{CO}_{2} & \text { and } & 85 \% & \mathrm{~N}_{2}\end{array}$ atmospheric conditions. They are obligately parasitic inhabitants of mucous membranes and remain infectious for only a few weeks outside their hosts. There are at least 37 known species and subspecies of this genus, with just one subset of clear pathogens. The zoonotic agents C. jejuni subsp. jejuni, C. coli, C. lari and C. upsaliensis are thermophilic species, that can grow at $42^{\circ} \mathrm{C}$. They may be directly or indirectly transmitted from other vertebrates to humans. There is sufficient evidence to believe that the risk of acquisition of $C$. jejuni increases due to the contact with infected dogs, particularly those with diarrhea (Marks et al. 2011; Percival 2014; Poxton et al. 2015).

\section{Clinico-pathological features}

In dogs, clinical signs of Campylobacteriosis are nonspecific and depend on the severity and duration of infection. Incubation period is often short, with symptoms of enteritis mostly develop within three days. The main sign is mild diarrhea with blood or mucus. Fever, vomiting, anorexia, and lethargy are less frequent signs. In young and physically weak animals, dehydration is more prevalent. Abnormally high fluid in colonic contents, as well as thickening, congestion, and edema of the colonic mucosa, are common macroscopic findings in both naturally and experimentally infected dogs. Microscopically, thickening of the mucosa is caused by epithelial glandular hyperplasia. Findings of subepithelial congestion, hemorrhage, as well as inflammatory cells infiltration have also been reported. The colon and cecum show diminished epithelial cell heights and brushed borders, with a reduction in the number of goblet cells. Staining samples with sliver staining to help detect the filamentous bacteria in intestinal crypts may be needed to confirm a diagnosis of Campylobacteriosis, as the pathological findings in many cases of Campylobacter infections are non-specific. Common extra-intestinal sequalae of Campylobacter infections in dogs include abortions, perinatal death, icterus, and other liver disorders.

In humans, the incubation period usually lasts from 3 to 5 days, with the eventual development of bloody or mucoid diarrhea in most cases. In $30 \%$ of patients, Campylobacteriosis may initially be manifested by an influenza-like symptoms rather than diarrhea. In such cases, the patients may complain fever, headache, weakness, dizziness, and myalgia. In immunosuppressed patients, extra-intestinal symptoms such as bacteremia, meningitis, and reactive arthritis can also be seen (Skirrow 2002; Marks and Kather 2003; Marks et al. 2011; Sahin et al. 2014; Kreling et al. 2020). 


\section{Diagnosis}

Reaching a definitive diagnosis of Campylobacteriosis in dogs is often challenging. This is largely due to high prevalence of colonization in healthy animals. Fecal cytology with a finding of curved, "Campylobacter-like" species is used by some workers, leading to possible falsepositive diagnoses. All Campylobacter spp. Are not pathogenic, and some other harmless bacteria morphologically resemble to Campylobacter. Consequently, fecal culturing has become the gold standard diagnostic test. Although difficult to interpret at time, fecal culturing has the benefit of supplying isolates for species identification and antibiotic sensitivity tests. It is essential to define the specific species of Campylobacter in order to reach a proper diagnosis. In particular, it is important to differentiate catalase-positive species $(C$. jejuni, $C$. coli) from catalase-negative ones, as a finding of catalase-positive Campylobacters may be clinically important. For the detection of $C$. jejuni and $C$. coli antigens, a rapid, simple, and inexpensive ELISA diagnostic tool, with an efficiency comparable to that of bacterial cultivation, has been developed. Several PCR tests have also been developed for the detection of different Campylobacter species. Although PCR tests may be more sensitive, yield faster results, and detect a wide range of species, it is not clear if their higher sensitivities would actually help in the diagnose of Campylobacteriosis or add to potential false-positive diagnoses (Marks and Kather 2003; Chaban et al. 2009; Chaban et al. 2010; Granato et al. 2010; Kaakoush et al. 2015).

Unlike canine infections, the lower prevalence of colonialization with Campylobacters in humans means that Campylobacteriosis can be more easily diagnosed. Campylobacter morphology and dart motility can be detected in feces by darkfield or phase-contrast microscopy. Culture is the most widely used tool in the detection of thermophilic and non-thermophilic species. Testing specifically for the thermophilic $C$. jejuni and $C$. coli should be sufficient for a diagnosis of Campylobacterosis in humans. Test for the non-thermophilic C. fetus, and $C$. upsaliensis, and other species with a variable growth capacity at $42^{\circ} \mathrm{C}$ may not be so helpful.

Moreover, the type of culture media used, especially for the detection of $C$. upsaliensis, may have a major influence on the accuracy of results, since $C$. upsaliensis is vulnerable to selective medium antimicrobials. Due to the fastidious nature of Campylobacter, single negative cultivation does not preclude Campylobacteriosis. In addition to PCR technologies, other molecular approaches used in recognizing or detecting Campylobacter organisms include random amplified polymorphic DNA, whole-genome sequencing and matrix-assisted laser desorption/ionization time-of-flight (MALDI-TOF) (Moore et al. 2005; Davis and DiRita 2008; Silva et al. 2016; Schürch et al. 2018).

\section{Therapeutic}

Treatment of Campylobacter infections in dogs is often conservative. For one thing, Campylobacter infections in dogs are invariably self-limiting. In addition, Campylobacter spp. isolated from dogs have demonstrated resistance to the commonly used antimicrobials, which would otherwise damage the normal intestinal microflora. Thus, supportive therapy constitutes the primary line of treatment, with the antimicrobial treatment given only if required. Treatment seems to be more focused at controlling clinical signs rather than at the clearance of the culprit bacteria. In young animals with moderate infections, or in patients with high fever, bloody or severe diarrhea, and patients who experience chronic infections, antimicrobials should be prescribed early. The best first-line drug options are macrolides (e.g., erythromycin). Other medications are often reserved for cases with erythromycin resistance or for refractory conditions. These include tetracyclines, fluoroquinolones, and chloramphenicol (Marks et al. 2011; Cho et al.-2014; Rodrigues et al. 2015; Weese et al. 2015). In human infections, management of Campylobacteriosis primarily involves the administration of fluid and electrolyte therapy. Antibiotics become necessary when symptoms persist. Effective antibiotic therapy should be initiated within three days of illness. Antibiotic drugs of choice for Campylobacteriosis treatment in humans include fluoroquinolones, aminoglycosides, tetracyclines, macrolides, beta-lactams, and erythromycin. However, erythromycin should be avoided in cases of extraintestinal infections caused mainly by $C$. fetus because of the prevalence of resistance to this drug. Ciprofloxacin, vancomycin, and quinolones are other good alternatives (Gilbert et al. 2007; Bardon et al. 2009; Guarino et al. 2014; Bolton 2015; Bruzzese et al. 2018).

\section{Pasteurellosis}

\section{Overview}

Pasteurellosis is a bacterial disease caused by organisms of the genus Pasteurella. It afflicts animals and is often transmitted to humans through bites or scratch injuries. Over six Pasteurella species as well as multiple serotypes exist. $P$. multocida and $P$. canis are typical dog isolates. $P$. multocida is the primary cause of human illness, with $P$. multocida subsp. multocida, P. canis, P. multocida subsp. septica, $P$. stomatis, and $P$. dagmatis subtypes cause infection in humans (Oehler et al. 2009).

\section{Etiology}

Pasteurella multocida belongs to the family Pasteurellaceae. It is a gram-negative, 0.3-1.2 $\mu \mathrm{m}$ long, non-motile, facultatively anaerobic coccobacillus, often with bipolar staining. It is usually found in the oral cavity and intestinal tracts of a number of animal species. This organism is an opportunistic pathogen that causes infection both in livestock and humans. Studies have shown $P$. multocida to be a part of natural mammalian mouth microbiota with colonization rates ranging from 50 to $66 \%$. Among dogs and humans, infection is commonly associated with intimate contact with a dog through 
sniffing, licking, or bed-sharing Lefebvre et al. 2006; GuetRevillet et al. 2013; Christenson et al. 2015).

\section{Clinico-pathological features}

Pasteurella infections, which can be primary or secondary in nature, are usually more progressive than most other bacterial infections. An infected dog may show a bite or scratch wound. Within 8-48 hours, soft tissue cellulitis, erythema, tenderness, swelling, focal abscesses with serosanguineous to purulent, malodorous, dark yellow discharges may develop around the site of the wound. More serious sequalae in the form of soft tissue infection, septicemia, tenosynovitis, septic arthritis, osteomyelitis, and meningitis may occur as a result of bacterial dissemination. Low-grade fever, typically associated with lymphadenopathy, may also be observed. The proliferation of Pasteurella species after viral infections induce an influx of inflammatory cells and cytokine mediators, resulting in upper and lower airways infection, including the typical Pasteurella fibrinopurulent pneumonia (Wiebe 2015).

The spectrum of human disease resulting from infection with $P$. multocida ranges from a mild, superficial affliction, with swelling, inflammation, and intense pain at the bite site a few hours after the exposure to more serious infections, both invasive and localized. The more serious infections may involve the oral cavity, respiratory tract, and/or soft tissue, leading to pharyngitis, sinusitis, meningitis, tracheobronchitis, pneumonia, empyema, and/or abscess formation (Bauerfeind and Krauss 2016).

\section{Diagnosis}

Since $P$. multocida is not a normal human inhabitant, a diagnosis of a $P$. multocida induced illness is comparatively uncomplicated. A sample taken from an infected site and examined under microscope reveals the presence of small gram-negative rods, often bipolar in shape. P. multocida grows best on media enriched with serum, blood, brain or heart infusion. Antigens are not commercially available as part of routine laboratory study kits, so antibodies cannot be detected in serum samples. Other diagnostic techniques, including PCR, capsule typing, and $16 \mathrm{~S}$ rRNA gene sequencing, have also been developed for the detection of Pasteurella multocida infection in humans (Bauerfeind and Krauss 2016).

\section{Therapeutics}

Penicillins are the antibiotic of choice for the treatment of dogs with Pasteurella infections. Localized infections are typically treated with an oral single-agent beta-lactam. For deep or severe disseminated infections, therapy may involve parenteral administration of penicillin, cefoxitin, and a carbapenem for 10-14 days. For the management of tenosynovitis, septic arthritis, osteomyelitis, or meningitis, however, 4-6 weeks of intravenous therapy is typically required.

In humans, a lack of culture findings would suggest the use of antibiotics with broad-spectrum coverage against
Staphylococci, Streptococci, and anaerobes, since infections with bites are mostly polymicrobial. Dog bite treatment typically requires penicillin as the antibiotic of choice, but most cases are more commonly treated with amoxicillin-clavulanate. Other alternatives include the use of second and third-generation cephalosporins (e.g., cefuroxime, cefpodoxime). Patients allergic to penicillin are usually treated with doxycycline or fluoroquinolones (Oehler et al. 2009;Wiebe 2015; Bauerfeind and Krauss 2016; Bennett et al. 2019).

\section{Bordetella infection (kennel cough)}

\section{Overview}

Bordetella infection, primarily caused by Bordetella bronchiseptica, is an opportunistic bacterial pathogen. Bordetella species, frequently involved in respiratory diseases in both animals and humans, are highly infectious and spread primarily by aerosolization among animals living under stressful conditions. In dogs, it is common in puppies with Canine Infectious Respiratory Disease Complex (CIRDC, "kennel cough"), causing rhinitis, tracheobronchitis, and pneumonia. It may affect humans who are immunosuppressed, leading to pneumonia and infections of the upper respiratory tract (Bhardwaj et al. 2013; Echeverri-Toro et al. 2015; Rampelotto et al. 2016).

\section{Etiology}

Bordetella is a motile, aerobic, gram-negative coccobacillus capable, colonizing the mucous membranes of the upper respiratory tract, causing specific damage to the respiratory epithelia of a large number of potential mammalian hosts. Such damage paves the way for super-infections with resident commensal bacteria, including Streptococcus spp., Mycoplasma spp., and Pasteurella spp. Such superinfections invariably worsen the prognosis and render decisions regarding the best antimicrobial treatment more complex. The most common cause of illness among Brodetella spp is $B$. pertussis, the leading causative agent of whopping cough in humans. The whopping cough may also result from $B$. parapertussis infection. A similar disease in dogs is caused by $B$. bronchiseptica, which can attack animals of all breeds and of all ages alongside humans. The incubation period of the infection ranges from 2 to 10 days. Shedding of the bacteria may continue for up to two months postinfection. The most common routes of transmission are direct contact with oral or nasal secretions or the inhalation of sneeze droplets contaminated with the infectious agent. Bordetella spp. can survive for a few hours in respiratory secretions, for 45 days in the soil, and for up to 24 weeks in lake water. Fomites or water sources contaminated with $B$. bronchiseptica may also serve as sources of infection (Parkhill et al. 2003; Weese and Fulford 2011; Wiebe 2015; Bauerfeind and Krauss 2016; Weese and Evason 2019). 


\section{Clinico-pathological features}

Infection with Bordetella is typically starts with the bacteria adhering to the cilia of respiratory tract epithelium with the help of several virulence factors. Once attached, ciliostasis, epithelial destruction with mucociliary clearance results in failure of the system, promoting further colonization and persistence of bacteria. After colonization, toxins released from $B$. bronchiseptica are responsible for local and systemic inflammatory damage. Following the incubation period, infected dogs start coughing. Initially, the cough is dry and paroxysmal, eventually becoming productive with serous to mucopurulent nasal discharge, conjunctivitis, and fever. Focal areas of epithelial degeneration, necrosis, and congestion in the lamina propria, infiltrated with macrophages and lymphocytes, may be seen on microscopic examination. In addition, mucopurulent exudates often accumulate in the lumen of the airways, and "carpet-like" bacterial clusters between the cilia of tracheobronchial epithelium can be seen. Infection with fever, lethargy, and productive cough can progress to bronchopneumonia and contribute to tracheal collapse. In some cases, the alveoli become filled with neutrophils and macrophages, while fibrin and erythrocytes are predominate in the alveolar spaces in other cases.

In humans, although $B$. bronchiseptica infections remain clinically rare, a pertussis-like disease caused by this species of Bordetella has been reported among immunocompromised individuals. Upper and lower respiratory tract infections may develop, with affliction ranging from an asymptomatic carriage or the development of mild sinusitis to severe infections such as bronchitis or necrotizing pneumonia; although occurrence of such infections is rare (Woolfrey and Moody 1991; Mattoo and Cherry 2005; Oskouizadeh et al. 2011; Wernli et al. 2011; Taha-Abdelaziz et al. 2016).

\section{Diagnosis}

In cases of suspected $B$. bronchiseptica infections, a variety of samples may be used for bacteriological culturing or conducting a modern PCR analysis. These include pharyngeal swabs, bronchoalveolar wash fluids, or transtracheal washings. Specimens obtained from the lower respiratory tract are more dependable relative to nasal or oropharyngeal swabs, as $B$. bronchiseptica has been frequently isolated from upper respiratory tracts of clinically healthy dogs. Moreover, prior vaccination with one of the newer bacterial intranasal vaccines and low bacterial numbers could yield both false PCR negative and positive results. Consequently, differentiating between the presence of $B$. bronchiseptica as part of the normal microbiota or as a cause of disease, interpretating test results, and deciding whether or not to administer treatment can be quite challenging (Chalker et al. 2003; Schulz et al. 2014; Viitanen et al. 2015).

\section{Therapeutics}

In both dogs and other animal species with uncomplicated conditions, the antimicrobial treatment seems unnecessary. Complete and quick recovery should not always be anticipated, as viral co-infections are common. Antimicrobials can shorten the course of the disease. Where necessary, antibiotic treatment should be based on culture and sensitivity, as some strains have shown resistance to both penicillin and sulfa drugs. The most frequently used antibiotics for the treatment of $B$. bronchiseptica infections include amoxicillin/clavulanic acid and cephalexin. Aminoglycosides appear to be particularly useful against $B$. bronchiseptica. The administration of aerosolized gentamicin, intravenous fluids, good nutrition, and extra oxygen can help treatment of severe infections when animals fail to respond to parenteral medication (Ford 2006; Rath et al. 2008; Vieson et al. 2012).

\section{Leptospirosis}

\section{Overview}

Leptospirosis is one of most widespread zoonotic waterborne bacterial diseases. It is caused by bacteria of the genus Leptospira. To date, more than 260 different Leptospira serovars have been discovered. They often cause hepatorenal disease with occasional affliction of other body systems. Nearly all mammals and marsupials can become renal carriers and cause human infection. Of all animals typically kept as pets, leptospirosis is quite frequent among dogs, especially those who spend time in forests or swampy areas. Dogs are natural carrier hosts. They are susceptible to infection with the $L$. canicola serovar as well as various other serovars and shed these pathogens in urine for a period of a few weeks. Humans become accidental hosts via direct contact with infected animals, by being licked by an infected dog, by petting it, or through contaminated soil or water. The risk of infection rises among veterinarians, farmers, sewerage and slaughterhouse workers (Bharti et al. 2003; LealCastellanos et al. 2003; Ko et al. 2009; Adler and de la Peña Moctezuma 2010; Nelson and Couto 2014).

\section{Etiology}

Leptospira spp., are a complex group of highly mobile, obligate aerobic, long and thin bacteria. They are spiralshaped with both ends bent in a hook-like fashion and measure about 6 to $20 \times 0.1 \mu \mathrm{m}$. Leptospira spp. are gramnegative bacteria with particular affinity for the urogenital tract, especially the kidneys. Disease outbreaks occur within 3 months of rainy weather. Excreted leptospira can survive for months in a humid environment but die very quickly under dry conditions. Dogs can become infected by drinking, swimming and wading in contaminated water sources or when exposed to livestock or rat urine. The Leptospira can be transmitted through mucous membranes, cuts or abrasions, or by the inhalation of aerosolized infected water. The leptospiremic phase lasts approximately for 7 days, after which rapid multiplication, coagulopathy, and vasculitis within organs can result in kidney and/or liver disorders or lead to leptospiral 
pulmonary hemorrhage syndrome (Kohn et al. 2010; Sykes et al. 2011; Weese and Fulford 2011; Weese and Evason 2019).

\section{Clinico-pathological features}

After seven days of incubation, leptospirosis manifestations in dogs usually differ based on the virulence and serovar type of the organism, prior health status of the host and the target organs infected. Leptospirosis can vary from subclinical form with mild, intermittent, unrecognized fever to acute hemolytic anemia, septicemia, and hepatorenal failure with uncontrollable vomiting and bloody diarrhea. Hemolytic anemia leads to icterus and a swollen yellowish liver. Microscopic findings typically include hepatic portal lymphocytic infiltration, splenic hemosiderosis and, occasionally, centrilobular hepatic cell necrosis (as a consequence of anemic anoxia). Subacute illness may either involve fever and jaundice or chronically mild and persistent nephritis. Microscopic examination of the kidneys reveals swelling of tubular epithelia caused by bile pigment and hemoglobin. Kidney-related symptoms such as polyuria/polydipsia and/or oliguria/anuria, vomiting, anorexia, and lethargy may extend from one month to one year after infection. The causative bacteria may remain in the kidney tubules causing chronic disease, evident microscopically in the renal parenchyma (most commonly in the cortex) in the form of greyish-white focal lesions with swollen, granular and vacuolated epithelium.

In humans, most patients are diagnosed on the basis of subclinical flu-like febrile disease after an incubation period of 7-12 days. In its initial febrile phase, which usually lasts for $4^{-7}$ days, fever, headache, myalgia, conjunctivitis, nausea, and vomiting are commonly noted. Jaundice and renal failure can start by the end of the first week in severe cases. Significant icterus with elevated bilirubin, usually accompanied by severe glomerulonephritis or interstitial nephritis, is typically detected by the third week (Levett 2001; Ellis, 2015; De Brito et al. 2018; Esteves et al. 2018).

\section{Diagnosis}

Canine leptospirosis can be diagnosed using various methods, including urine examination with darkfield microscopy, blood and urine microbiological analysis, renal tissue histopathology, serology, and PCR of the blood, urine, and tissue. Currently, microagglutination tests (MAT) are the gold standard for serologic diagnosis. A diagnosis of leptospirosis entails that, over three weeks, the titer to at least one serovar increases four-times in early phase or decreases four-times in late phase. Modern molecular techniques such as PCR and immunoblotting can, in contrast to culture, reduce diagnostic time because culture requires special media, specific conditions, and a period of up to 16 weeks to reach a diagnosis. Traditional PCR-testing remains less sensitive than serological testing, often producing numerous false-negative results. Recently, improved techniques, such as multi-locus sequence typing (MLST) that work by analyzing the bacterial genome or its specific regions for the typing of Leptospira strains, have been developed (Miller et al. 2011; Koizumi et al. 2015; Schuller et al. 2015; Miotto et al. 2018; Troìa et al. 2018).

For diagnostic purposes, human leptospirosis requires detailed and comprehensive case history, including a review of occupational and outdoor activities, animal contacts, and the occurrence of sudden or recurrent fever. During the first ten days of illness, different visualization methods, such as darkfield microscopy, light microscopy on the silver stain, and immunostaining preparations, can be used to visualize Leptospira in blood or urine smears. However, the sensitivity of visualization methods is limited, and several samples must be taken due to the intermittent nature of leptospiral shedding in the urine. Microbiological and serological techniques are typically used to confirm the diagnosis. The Microagglutination Test (MAT) and an Indirect Hemagglutination Test (IHA) are both designed for the diagnosis of human illness, as well as to detect recent dog infections. In contrast with MAT and ELISA, the IHA is specific and sensitive and is easy to perform (Vijayachari and Sehgal 2006; American Academy of Pediatrics and Pickering 2009).

\section{Therapeutics}

Canine Leptospirosis antimicrobial therapy is mandatory, irrespective of the severity of the disease, because of the associated public health risk. Shedding of viable Leptospira should end within 24-48 hours after the commencement of treatment. Leptospira spp. are highly susceptible to both penicillin $G$ and doxycycline, which are essential to the mitigation of leptospiremia and the prevention of further organ damage. To these ends, they are most efficacious when given in early phase of the disease in both dogs and humans. Doxycycline is the drug of choice for the treatment of renal infection in animals with serious renal impairment but minimal hepatic dysfunction. Parenteral penicillin G or ampicillin can be used as alternatives, but both are less effective in removing organisms from the kidney.

In severe Leptospirosis patients, intravenous penicillin $G$, cephalosporin of third-generation, or erythromycin can be prescribed in severe cases. More recently, azithromycin, cefotaxime, ceftriaxone, and fluoroquinolones have been shown to have comparable potency to doxycycline against multiple serovars in vitro. Intense care unit admission is often vital for patients with icteric Leptospirosis because multiple organ involvement and decompensation can rapidly occur. Corticosteroids may help, but their use iscontroversial for the treatment of patients with renal impairment (Greene 2012; Jiménez et al. 2018; Wang et al. 2020).

\section{Brucellosis}

\section{Overview}

Brucellosis is an infectious disease of humans and animals caused by bacteria of the genus Brucella. In humans, 
Brucellosis is mostly a foodborne disease, caused by Brucella melitensis through consuming unpasteurized milk or its products. In dogs, Brucellosis is most frequently a reproductive disease caused by $B$. canis, a zoonotic pathogen that is the major cause of diskospondylitis. Brucella canis, however, is the least frequent cause of human Brucellosis. Dogs can be infected by several Brucella species, including B. abortus and $B$. melitensis, and contribute to the spreading of these organisms in farms, potentially becoming a source of human infection (Weese and Fulford 2011; Bennett et al. 2019).

\section{Etiology}

B. canis is a small (1.o to $1.5 \mu \mathrm{m}$ ), gram-negative, coccoid to short rod-shaped, non-motile, obligately parasite, with a moderate ability to survive outside the host. Differences in biochemical and antigenic reactions distinguish $B$. canis from other members of the genus Brucella. In domestic and wild canids, $B$. canis infects a susceptible canine host by penetrating the mucous membranes, especially those of the oral cavity, vagina, and conjunctiva. B. canis usually harbors in the lymph nodes of gastrointestinal tracts for extended periods and then transmitted primarily by ingestion or inhalation of aerosolized post-abortion material. Venereal transmission of Brucellosis has also been reported (Lucero et al. 2010; Atluri et al. 2011; Von Bargen et al. 2012; Galinska and Zagorski 2013).

\section{Clinico-pathological features}

Acute lymphadenitis is caused by intracellular proliferation of organisms within histiocytes and macrophages residing in the regional lymph nodes. Passage of the organisms into the blood stream leads to bacteremia. Organisms may then localize in male or female reproductive organs, the placenta, fetus, or udder. They may also localize in distant lymph nodes, the spleen, liver, joints, or bone, resulting in varied signs. In dogs, $B$. canis infection is characterized by prolonged bacteremia, reproductive failure, and infertility as a result of abnormal spermatogenesis in males and abnormal oogenesis in females. In pregnant bitches, $B$. canis colonizes in placental epithelial cells, inducing placentitis and abortion at late-term (weeks 7-9). Macroscopically, necrosis of the cotyledons appears dull and granular. The intercotyledonary chorion becomes edematous and full of brownish exudate. Many organisms in the chorionic epithelial cells are seen microscopically. Puppies infected in utero do not usually survive to weaning. In male dogs, B. canis causes orchitis, epididymitis and prostatitis. Complications such as lymphadenopathy, splenomegaly, discospondylitis, uveitis, meningitis, glomerular nephritis, and dermatitis can also occur periodically.

In humans, symptoms and signs of $B$. canis infection are, for the most part, unspecific and may vary from an undulant or persistent fever, splenomegaly, malaise, myalgia, headaches, and anorexia, to symptoms of more serious conditions such as endocarditis, osteomyelitis and septicemia (Wanke 2004; Lucero et al. 2005a, Hollett 2006; Olivera and Di-Lorenzo 2009; Lucero et al. 2010).

\section{Diagnosis}

Isolation of $B$. canis through culturing is the gold standard diagnostic test. Appropriate sites for sample-taking include the placenta, lymph nodes, prostate and spleen. Direct Köster stain microscopy guarantees rapid orientation, and blood cultures are typically effective within the first eight weeks after infection. The sensitivity of blood cultures is reduced with time, as bacteria are most frequently isolated following acute, not chronic, infections. Compared with blood culture, the diagnostic sensitivity of whole blood PCR is far superior. It stands at $100 \%$ in naturally infected dogs and is often used to confirm the species of the Brucella isolates. B. canisspecific serological testing may also be used, and a definitive diagnosis can be made from a positive result in combination with consistent clinical signs. $B$. canis total antibody screening is performed using a rapid slide agglutination test with 2-mercaptoethanol. A confirmatory indirect ELISA test should then be conducted in order to detect antigen-specific IgG or IgM antibodies (Lucero et al. 2005b; Sánchez-Jiménez et al. 2014).

\section{Therapeutics}

For Brucellosis, the general recommendation is to avoid medication and to euthanize truly infected animals because of the threat to both canine and human populations. Traditionally, tetracyclines were most commonly used in the treatment of infections. Monotherapy, however, has been associated with high relapse rates. Combination therapies that include rifampin or streptomycin appear to have the best efficacy. Neutering, along with enrofloxacin therapy for a minimum one-month period are needed because of the intracellular nature of causative organisms and the inability of some pharmaceutical products to penetrate certain areas of the canine body.

For humans, typical antibiotic therapy comprises oral doxycycline and rifampin for a period of 6 to 8 weeks, to which a one-to-two-week course of aminoglycosides is usually added. In children below eight years of age and pregnant mothers, where tetracyclines are contraindicated, a trimethoprim-sulfamethoxazole mixture and rifampin or an aminoglycoside should be prescribed (Hollett 2006; Wanke et al. 2006; Sykes 2013; Wiebe 2015).

\section{Lyme borreliosis}

\section{Overview}

Lyme disease (borreliosis) is a multisystemic, tickvectored, zoonotic disease caused by the bacterium Borrelia burgdorferi, which is transmitted by Ixodes spp. It may be manifested with any of a large number of 
dermatological, rheumatological, neurological and cardiac symptoms in both humans and dogs. Dogs have been identified as a competent reservoir of $B$. burgdorferi among pet animals, serving as carriers of infected ticks from wildlife hosts to human settings. Dogs have also been proposed as a 'sentinel animal' giving advanced warning of possible human exposure to infected ticks and helping detect emerging risk areas of Lyme disease, as dogs and humans mostly live in and share the same environment and visit the same outdoor areas (Duncan et al. 2004; Mead et al. 2011; Otranto et al. 2015; Steere et al. 2016; DeLong et al. 2019).

\section{Etiology}

The Borrelia burgdorferi sensu lato complex is a representing term for all genospecies of $B$. burgdorferi that cause Lyme disease. Borreliae are small, thin, elongated spiral-shaped, commonly spirochetal-structured bacteria, $0.2 \mu \mathrm{m}$ to $30 \mu \mathrm{m}$ in size. They cannot survive as free-living organisms in the environment and are transmitted between vertebrate reservoir hosts and tick vectors. The main reservoir hosts are small mammals. Dead-end hosts are sheep, dogs, and humans. Naive ticks that feed on infected dogs invariably become infected. These ticks would then infect other vertebrates, including humans. By introducing infected ticks into the human population, dogs raise the level of dog owners' exposure to $B$. burgdorferi (Fritz and Kjemtrup 2003; Greene 2012; Parry 2016).

\section{Clinico-pathological features}

Clinical Lyme disease is a less frequent consequence of an infection in dogs than in humans; over $95 \%$ of naturally infected dogs are asymptomatic. Others may show fever, lethargy, decreased appetite, lymphadenopathy, lameness, and/or a form of renal disorder. Shifting limb lameness is a common manifestation of Lyme disease in dogs. It results from joint swelling coupled with fibrin and neutrophils effusion, occurring in $9-28 \%$ of the seropositive dogs. In most cases, lameness starts in the limb nearest the tick bite spot, improves and resolves within two days then possibly remanifesting in the other limb. Skin biopsy samples typically show superficial perivascular lymphoplasmacytic infiltrates with mast cell accumulations. Lameness can progress to nonsuppurative, predominantly lymphoplasmacytic inflammation, leading to chronic non-erosive polyarthritis if the infection remains undiagnosed and untreated. Lyme nephritis is an uncommon but extreme type of severe protein-losing nephropathy with renal failure. It is typically seen in less than $2 \%$ of seropositive dogs. Renal lesions typically include glomerulitis, diffuse tubular necrosis with regeneration, and interstitial inflammation. Dogs with Lyme nephritis can become dehydrated, suffer from edema, pleural effusion, ascites, elevated blood pressure, neurological, cardiac, and retinal problems (Krupka and Straubinger 2010; Greene 2012; Leschnik 2014).
Lyme disease may also cause serious complications in humans. A typical human infection starts with an expanding skin lesion termed erythema migrans. This socalled erythema migrans is a red spread out rash that may or may not show central clearing. It is often accompanied by signs of weakness, fever, headache, mild stiff neck, arthralgia, or myalgia, which, if untreated, can be followed by early disseminated infection, with the development of neurologic or cardiac abnormalities. Moreover, an untreated infection may progress to cause arthritis, peripheral neuropathy, or encephalopathy. These longterm sequelae can take place over variable periods, ranging from one week to a few years (Steere and Angelis 2006; Steere and Sikand 2003; Wormser 2006; Ray et al. 2013; Steere et al. 2016).

\section{Diagnosis}

In dogs, diagnosis of the Lyme disease is based on clinical symptoms, history of exposure, and positive antibody response. Recently developed methods of diagnosis, including the C6 peptide test for Lyme, as well as serology and PCR testing, could be performed. A complete blood picture may demonstrate mild to moderate thrombocytopenia and leukocytosis. Azotemia, moderate to pronounced hypoalbuminemia, metabolic acidosis, and electrolyte changes can be present in dogs with renal disease. Urinalysis may show isosthenuria, proteinuria, pyuria, and hematuria. In such cases, a renal biopsy would identify the affliction as an immune-complex disorder but would not confirm a case of Lyme nephritis (Wiebe 2015; Parry 2016).

Strategies for the diagnosis of Lyme disease in humans vary. With the exception of cases with pathognomonic erythema migrans, the diagnosis of Lyme Borreliosis usually requires confirmation by means of a microbiological, serological, or molecular diagnostic assay. $B$. burgdorferi can be cultured from erythema migrans skin lesions, but such testing is not consistently available. In early disseminated Lyme Borreliosis patients, $B$. burgdorferi are typically cultured from blood samples. In patients with early disseminated disease, clinical severity predicts the likelihood of hematogenous disease. Serological diagnosis can be difficult, especially in early cases, because IgM is typically not detectable within the first 1-2 weeks following infection and IgG does not often appear for 4-6 weeks. In addition, certain patients with solitary erythema migrans may never show seroconversion.

Serological testing is further limited by the fact that antibodies could last in the blood stream for many years post-infection and thus cannot be used to determine the effectiveness of antibiotic treatment. In recent years, commercial serological tests for the diagnosis of Borrelia infections have been developed. Among these is the ELISA-based TickPlex assay, which incorporates a new antigen from the round body/persister forms of Borrelia. This assay was shown to be useful at different Lyme Borreliosis stages, and the improved test can concurrently assess IgM and IgG antibodies of various bacterial and 
viral tick-transmitted pathogens. B. burgdorferi DNA has been successfully detected by PCR in synovial fluid and less definitively in cerebro-spinal fluid (CSF). Due to lack of standardization, Lyme Borreliosis PCR testing is not considered part of routine clinical practice. There is a continuous interest in novel approaches to diagnostics, including Borrelial antigen identification, nucleic acid amplification, and genomic sequencing (Wormser et al., 2005; Li et al. 2011; Steere et al. 2016; Wormser et al. 2017; Branda et al. 2018; Trevisan et al. 2020).

\section{Therapeutics}

Management of canine cases of Lyme disease is multifaceted. Dogs require routine medication for cases of protein-losing nephropathy. Treatment of dogs also necessitates long-term administration of antimicrobials, such as cephalosporins, amoxicillin, doxycycline, or azithromycin. Immunosuppression therapy may also be required. If animals do not tolerate doxycycline, oral cefuroxime axetil is an effective alternative in early Lyme disease cases (Fritz and Kjemtrup 2003; Krupka and Straubinger 2010; Wiebe 2015).

The aim of antibiotic therapy for early human Lyme disease, whether local or disseminated, is to shorten the duration of erythema migrans and symptoms associated with them and to prevent the development of the latestage disease. Efficient antibiotic treatment with oral doxycycline and amoxicillin is effective in most cases. Cefuroxime axetil is an option for those patients who cannot be treated with doxycycline or amoxicillin. Azithromycin, clarithromycin, and erythromycin can also be used, but may be less effective (Sanchez et al. 2016; Steere et al. 2016).

\section{REFERENCES}

Adak GA et al., 2005. Disease risks from foods, England and Wales, 1996-2000. Emerging Infectious Diseases 11: $365-372$.

Adler B and de la Peña Moctezuma A, 2010. Leptospira and leptospirosis. Veterinary Microbiology 140: 287296.

American Academy of Pediatrics and Pickering LK, 2009. Red Book: Report of the Committee on Infectious Diseases. $28^{\text {th }} \mathrm{Ed}$.

Atluri VL et al., 2011. Interactions of the human pathogenic Brucella species with their hosts. Annual Review of Microbiology 65: 523-541.

Bardon J et al., 2009. Prevalence of Campylobacter jejuni and its resistance to antibiotics in poultry in the Czech Republic. Zoonoses Public Health 56: 111-116.

Bauerfeind R and Krauss H, 2016. Zoonoses: Infectious Diseases Transmissible from Animals to Humans, 4th Ed. Washington, DC: ASM Press.

Bennett JE et al., 2019. Mandell, Douglas, and Bennett's Principles and Practice of Infectious Diseases E-Book. $9^{\text {th }}$ Ed. Elsevier Health Sciences

Bhardwaj et al., 2013. Bordetella bronchiseptica infection and Kennel cough in dogs. Advances in Animal and
Veterinary Sciences 1: 1-4.

Bharti et al., 2003. Leptospirosis: a zoonotic disease of global importance. The Lancet Infectious Diseases 3: 757-771.

Bolton DJ, 2015. Campylobacter virulence and survival factors. Food Microbiology 48: 99-108.

Branda et al., 2018. Advances in serodiagnostic testing for Lyme disease are at hand. Clinical Infectious Diseases 66: 1133-1139.

Bruzzese et al., 2018. Antibiotic treatment of acute gastroenteritis in children. F1ooo Research 7: 193.

Chaban et al., 2009. Development of cpn6o-based realtime quantitative PCR assays for the detection of 14 Campylobacter species and application to screening of canine fecal samples. Applied and Environmental Microbiology 75:3055-3061.

Chaban et al., 2010. Detection and quantification of 14 Campylobacter species in pet dogs reveals an increase in species richness in feces of diarrheic animals. BMC Microbiology 10: 73.

Chalker et al., 2003. Respiratory disease in kennelled dogs: serological responses to Bordetella bronchiseptica lipopolysaccharide do not correlate with bacterial isolation or clinical respiratory symptoms. Clinical and Diagnostic Laboratory Immunology 10: 352-356.

Cho et al., 2014. Characterization of antimicrobial resistance and application of RFLP for epidemiological monitoring of thermophilic Campylobacter spp. isolated from dogs and humans in Korea. Korean Journal of Veterinary Research 54: 91-99.

Christenson et al., 2015. Pasteurella multocida infection in solid organ transplantation. The Lancet Infectious Diseases 15: 235-240.

Davis L and DiRita V, 2008. Growth and laboratory maintenance of Campylobacter jejuni. Current Protocols in Microbiology doi: 10.1002/9780471729259. mco8aoisio

De Brito et al., 2018. Pathology and pathogenesis of human leptospirosis: a commented review. Revista do Instituto de Medicina Tropical de São Paulo 6o: 23-32.

DeLong et al., 2019. Estimation of cumulative number of post-treatment Lyme disease cases in the US, 2016 and 2020. BMC Public Health 19: 352-359.

Duncan et al., 2004. The dog as a sentinel for human infection: prevalence of Borrelia burgdorferi C6 antibodies in dogs from southeastern and midAtlantic states. Vector-Borne and Zoonotic Diseases 4: 221-229.

Echeverri-Toro et al., 2015. Bordetella bronchiseptica recurrent bacteraemia in a patient with bone marrow transplantation. Biomédica 35: 302-305.

Ellis WA, 2015. Animal Leptospirosis. In: Leptospira and Leptospirosis. Springer 99-137.

Esteves et al., 2018. Diagnosis of human Leptospirosis in a clinical setting: Real-time PCR high resolution melting analysis for detection of Leptospira at the onset of disease. Scientific Reports 8: 9213-9222.

Finley et al., 2007. The risk of salmonellae shedding by dogs fed Salmonella-contaminated commercial raw 
food diets. The Canadian Veterinary Journal 48: 6975 .

Ford RB, 2006. Canine infectious tracheobronchitis. Infectious Diseases of The Dog and Cat. $4^{\text {th }}$ Edition Saunders Elsevier, St. Louis (MO) 2013: 55-65.

Fritz CL and Kjemtrup AM, 2003. Lyme borreliosis. Journal of the American Veterinary Medical Association 223: 1261-1270.

Galinska EM and Zagorski J, 2013. Brucellosis in humans etiology, diagnostics, clinical forms. Annals of Agricultural and Environmental Medicine 20(2): 233238.

Gilbert et al., 2007. The Sanford Guide to Antimicrobial Therapy. $-37^{\text {th }}$ Ed.-Vienna, Va: Antimicrobial Therapy.

Granato et al., 2010. Comparison of premier CAMPY enzyme immunoassay (EIA), ProSpecT Campylobacter EIA, and ImmunoCard STAT! CAMPY tests with culture for laboratory diagnosis of Campylobacter enteric infections. Journal of Clinical Microbiology 48 : 4022-4027.

Greene CE, 2012. Infectious Diseases of the Dog and Cat. Elsevier/Saunders.

Guarino et al., 2014. European Society for Pediatric Gastroenterology, Hepatology, and Nutrition/ European Society for Pediatric Infectious Diseases evidence-based guidelines for the management of acute gastroenteritis in children in Europe: update 2014. Journal of Pediatric Gastroenterology and Nutrition 59: 132-152.

Guet-Revillet et al., 2013. Paediatric epidemiology of Pasteurella multocida meningitis in France and review of the literature. European Journal of Clinical Microbiology and Infectious Diseases 32: 1111-1120.

Hollett RB, 2006. Canine Brucellosis: Outbreaks and compliance. Theriogenology 66: $575^{-587}$.

Jiménez et al., 2018. Leptospirosis: Report from the task force on tropical diseases by the World Federation of Societies of Intensive and Critical Care Medicine. Journal of Critical Care 43: 361-365.

Joffe DJ and Schlesinger DP, 2002. Preliminary assessment of the risk of Salmonella infection in dogs fed raw chicken diets. The Canadian Veterinary Journal 43: 441-442.

Kaakoush et al., 2015. Global epidemiology of Campylobacter infection. Clinical Microbiology Reviews 28: 687-720.

Ko et al., 2009. Leptospira: the dawn of the molecular genetics era for an emerging zoonotic pathogen. Nature Reviews Microbiology 7: 736-747.

Koene et al., 2004. Simultaneous presence of multiple Campylobacter species in dogs. Journal of Clinical Microbiology 42: 819-821.

Kohn et al., 2010. Pulmonary abnormalities in dogs with Leptospirosis. Journal of Veterinary Internal Medicine 24: 1277-1282.

Koizumi et al., 2015. Multiple-locus variable-number tandem repeat analysis and clinical characterization of Leptospira interrogans canine isolates. Journal of Medical Microbiology 64: 288-294.

Kreling et al., 2020. Campylobacter sp.: Pathogenicity factors and prevention methods-new molecular targets for innovative antivirulence drugs? Applied Microbiology and Biotechnology 104: 10409-10436.

Krupka I and Straubinger RK, 2010. Lyme borreliosis in dogs and cats: background, diagnosis, treatment and prevention of infections with Borrelia burgdorferi sensu stricto. Veterinary Clinics: Small Animal Practice 40: 1103-1119.

Leal-Castellanos et al., 2003. Risk factors and the prevalence of Leptospirosis infection in a rural community of Chiapas, Mexico. Epidemiology and Infection 131: 1149-1156.

Lefebvre et al., 2006. Prevalence of zoonotic agents in dogs visiting hospitalized people in Ontario: implications for infection control. Journal of Hospital Infection 62: 458-466.

Lefebvre et al., 2008. Evaluation of the risks of shedding Salmonellae and other potential pathogens by therapy dogs fed raw diets in Ontario and Alberta. Zoonoses and Public Health 55: 470-480.

Leonard F, 2014. Salmonella infection and carriage: the importance of dogs and their owners. Veterinary Record 174: 92-93.

Leschnik M, 2014. Canine borreliosis: are we facing the facts? Veterinary Journal 199: 197-198.

Levett PN, 2001. Leptospirosis. Clinical Microbiology Reviews 14: 296-326.

Li et al., 2011. Burden and viability of Borrelia burgdorferi in skin and joints of patients with erythema migrans or lyme arthritis. Arthritis and Rheumatism 63: 22382247.

Lucero et al., 2005a. Unusual clinical presentation of Brucellosis caused by Brucella canis. Journal of Medical Microbiology 54: 505-508.

Lucero et al., 2005b. Diagnosis of human brucellosis caused by Brucella canis. Journal of Medical Microbiology 54: 457-461.

Lucero et al., 2010. Human Brucella canis outbreak linked to infection in dogs. Epidemiology and Infection 138: 280-285.

Macpherson et al., 2013. Dogs, zoonoses, and public health. CABI: Wallingford, Oxfordshire; Boston, MA.

Marks SL and Kather EJ, 2003. Bacterial-associated diarrhea in the dog: a critical appraisal. The Veterinary Clinics of North America. Small Animal Practice 33: 1029-1060.

Marks et al., 2011. Enteropathogenic bacteria in dogs and cats: diagnosis, epidemiology, treatment, and control. Journal of Veterinary Internal Medicine 25: 1195-1208.

Mattoo S and Cherry JD, 2005. Molecular pathogenesis, epidemiology, and clinical manifestations of respiratory infections due to Bordetella pertussis and other Bordetella subspecies. Clinical Microbiology Reviews 18: 326-382.

Mazick et al., 2006. An outbreak of Campylobacter jejuni associated with consumption of chicken, Copenhagen, 2005. Eurosurveillance 11: 137-139.

Mead et al., 2011. Canine serology as adjunct to human Lyme disease surveillance. Emerging Infectious Diseases 17: 1710-1712. 
Miller et al., 2011. Variability in results of the microscopic agglutination test in dogs with clinical Leptospirosis and dogs vaccinated against Leptospirosis. Journal of Veterinary Internal Medicine 25: 426-432.

Miotto et al., 2018. Diagnosis of acute canine Leptospirosis using multiple laboratory tests and characterization of the isolated strains. BMC Veterinary Research 14: 222-230.

Moore et al., 2005. Campylobacter. Veterinary Research 36: $351-382$.

Morley et al., 2006. Evaluation of the association between feeding raw meat and Salmonella enterica infections at a Greyhound breeding facility. Journal of the American Veterinary Medical Association 228: 15241532.

Nelson RW and Couto CG, 2014. Small Animal Internal Medicine. E-Book. Elsevier Health Sciences.

Oehler et al., 2009. Bite-related and septic syndromes caused by cats and dogs. The Lancet Infectious Diseases. 9(7): 439-447.

Olivera M and Di-Lorenzo C, 2009. Aislamiento de Brucella canis en un humano conviviente con caninos infectados. Colombia Médica 40: 218-220.

O'Neil J, 2018. Zoonotic infections from common household pets. Journal for Nurse Practitioners 14: 363-370.

Oskouizadeh et al., 2011. Isolation of Bordetella bronchiseptica in a dog with tracheal collapse. Comparative Clinical Pathology 20: 527-529.

Otranto et al., 2015. The role of wild canids and felids in spreading parasites to dogs and cats in Europe. Part I: Protozoa and tick-borne agents. Veterinary Parasitology 213: 12-23.

Parkhill et al., 2003. Comparative analysis of the genome sequences of Bordetella pertussis, Bordetella parapertussis and Bordetella bronchiseptica. Nature Genetics 35: 32-40.

Parry N, 2016. Canine Borreliosis: epidemiology, pathogenesis, clinical signs, and diagnostics. Companion Animal 21: 323-331.

Percival et al., 2014. Microbiology of Water-borne Diseases: microbiological aspects and risks. Elsevier, Academic Press: Amsterdam.

Poxton I et al., 2015. Molecular Medical Microbiology. Three-Volume Set. $1^{\text {st }}$ Edition Academic Prsss.

Rampelotto et al., 2016. Pneumonia caused by Bordetella bronchiseptica in two HIV-positive patients. Sao Paulo Medical Journal 134: 268-272.

Rath et al., 2008. Persistent Bordetella bronchiseptica pneumonia in an immunocompetent infant and genetic comparison of clinical isolates with kennel cough vaccine strains. Clinical Infectious Diseases 46: 905-908.

Ray et al., 2013. Three sudden cardiac deaths associated with Lyme carditis - United States, November 2012July 2013. MMWR Morbidity and Mortality Weekly Report 62: 993-996.

Rodrigues et al., 2015. Occurrence and characterization of Campylobacter spp. isolates in dogs, cats and children. Pesquisa Veterinária Brasileira 35: 365-370.
Rossi et al., 2008. Occurrence and species level diagnostics of Campylobacter spp., enteric Helicobacter spp. and Anaerobiospirillum spp. in healthy and diarrheic dogs and cats. Veterinary Microbiology 129: 304-314.

Sahin et al., 2014. Campylobacter jejuni as a cause of canine abortions in the United States. Journal of Veterinary Diagnostic Investigation 26: 699-704.

Sanchez et al., 2016. Diagnosis, treatment, and prevention of Lyme disease, human granulocytic Anaplasmosis, and Babesiosis: A Review. Journal of American Medical Association 315: 1767-1777.

Sánchez-Jiménez et al., 2014. Application of a polymerase chain reaction test for the detection of Brucella canis from clinical samples of canines and humans. Revista Colombiana de Ciencias Pecuarias 27: 3-11.

Schuller et al., 2015. European consensus statement on Leptospirosis in dogs and cats. Journal of Small Animal Practice 56: 159-179.

Schulz et al., 2014. Detection of respiratory viruses and Bordetella bronchiseptica in dogs with acute respiratory tract infections. Veterinary Journal 201: 365-369.

Schürch et al., 2018. Whole genome sequencing options for bacterial strain typing and epidemiologic analysis based on single nucleotide polymorphism versus gene-by-gene-based approaches. Clinical Microbiology and Infection 24: 350-354.

Silva et al., 2016. Campylobacter species isolated from poultry and humans, and their analysis using PFGE in southern Brazil. International Journal of Food Microbiology 217: 189-194.

Singhal et al., 2015. MALDI-TOF mass spectrometry: an emerging technology for microbial identification and diagnosis. Frontiers of Microbiology 6: 791-8o6.

Skirrow M, 2002. Campylobacter jejuni. Infections of the Gastrointestinal Tract pp: 719-740.

Steere AC and Angelis SM, 2006. Therapy for Lyme arthritis: strategies for the treatment of antibioticrefractory arthritis. Arthritis and Rheumatism 54: 3079-3086.

Steere AC and Sikand VK, 2003. The presenting manifestations of Lyme disease and the outcomes of treatment. The New England Journal of Medicine 348: 2472-2474.

Steere et al., 2016. Lyme borreliosis. Nature Reviews Disease Primers 2(1): 1-9.

Sykes JE, 2013. Canine and Feline Infectious Diseases - EBook. Elsevier Health Sciences.

Sykes et al., 2011. 2010 ACVIM Small Animal Consensus Statement on Leptospirosis: Diagnosis, Epidemiology, Treatment, and Prevention. Journal of Veterinary Internal Medicine 25: 1-13.

Taha-Abdelaziz et al., 2016. Cilia-associated bacteria in fatal Bordetella bronchiseptica pneumonia of dogs and cats. Journal of Veterinary Diagnostic Investigation 28: $369-376$.

Tenkate TD and Stafford RJ, 2001. Risk factors for Campylobacter infection in infants and young children: a matched case-control study. Epidemiology and Infection 127: 399-404. 
Trevisan et al., 2020. A practical approach to the diagnosis of Lyme Borreliosis: From clinical heterogeneity to laboratory methods. Frontiers in Medicine 7: 265- 279.

Troìa et al., 2018. Prospective evaluation of rapid point-ofcare tests for the diagnosis of acute Leptospirosis in dogs. The Veterinary Journal 237: 37-42.

Tsai et al., 2007. Salmonellae and campylobacters in household and stray dogs in northern Taiwan. Veterinary Research Communication 31: 931-939.

Vieson et al., 2012. A review of the pathology and treatment of canine respiratory infections. Veterinary Medicine: Research and Reports 3: 25-39.

Viitanen et al., 2015. Co-infections with respiratory viruses in dogs with bacterial pneumonia. Journal of Veterinary Internal Medicine 29: 544-551.

Vijayachari P and Sehgal SC, 2006. Recent advances in the laboratory diagnosis of Leptospirosis and characterisation of leptospires. Indian Journal of Medical Microbiology 24: 320-322.

Von Bargen et al., 2012. Internal affairs: investigating the Brucella intracellular lifestyle. FEMS Microbiology Reviews 36: 533-562.

Wang et al., 2020. Leptospirosis. In: StatPearls. StatPearls Publishing: Treasure Island (FL).

Wanke MM, 2004. Canine Brucellosis. Animal Reproduction Science 82-83: 195-207.

Wanke et al., 2006. Use of enrofloxacin in the treatment of canine Brucellosis in a dog kennel (clinical trial). Theriogenology 66: 1573-1578.
Weese JS and Evason M, 2019. Infectious diseases of the dog and cat: a Color Handbook. CRC Press: Boca Raton, Florida, USA.

Weese JS and Fulford MB, 2011. Companion Animal Zoonoses. Wiley-Blackwell: Ames, Iowa, USA.

Weese et al., 2015. ACVIM consensus statement on therapeutic antimicrobial use in animals and antimicrobial resistance. Journal of Veterinary Internal Medicine 29: 487-498.

Wernli et al., 2011. Evaluation of eight cases of confirmed Bordetella bronchiseptica infection and colonization over a 15-year period. Clinical Microbiology and Infection 17: 201-203.

Wiebe VJ, 2015. Drug Therapy for Infectious Diseases of the Dog and Cat. Hoboken, NJ (ed): John Wiley \& Sons, Inc.

Woolfrey BF and Moody JA, 1991. Human infections associated with Bordetella bronchiseptica. Clinical Microbiology Reviews 4: 243-255.

Wormser GP, 2006. Clinical practice. Early Lyme disease. The New England Journal of Medicine 354: 2794-2801.

Wormser et al., 2005. Brief communication: hematogenous dissemination in early Lyme disease. Annals of Internal Medicine 142: 751-755.

Wormser et al., 2017. Studies that report unexpected positive blood cultures for Lyme Borrelia - are they valid? Diagnostic Microbiology and Infectious Diseases 89: 178-181. 